Research Article

\title{
Vibration Performance Redistribution in Heterogeneous Networks
}

\author{
Genling Huang $\mathbb{D}^{1}$ and Jiqiang Wang $\mathbb{D}^{2}$ \\ ${ }^{1}$ Henan High-speed Railway Operation and Maintenance Engineering Research Center, \\ Zhengzhou Railway Vocational and Technical College, 56 Pengcheng Avenue, Zhengzhou 451460, China \\ ${ }^{2}$ Jiangsu Province Key Laboratory of Aerospace Power Systems, College of Energy and Power Engineering, \\ Nanjing University of Aeronautics and Astronautics, 29 Yudao Street, Nanjing 210016, China \\ Correspondence should be addressed to Jiqiang Wang; jiqiang_wang@hotmail.com
}

Received 10 July 2020; Revised 13 December 2020; Accepted 14 December 2020; Published 22 December 2020

Academic Editor: Antonio Batista

Copyright ( 92020 Genling Huang and Jiqiang Wang. This is an open access article distributed under the Creative Commons Attribution License, which permits unrestricted use, distribution, and reproduction in any medium, provided the original work is properly cited.

\begin{abstract}
Collective behaviors such as synchronization, consensus, and flocking have been extensively investigated over the past decades. Many important results have been disseminated concerning the properties of complex networks. Recent technological development requires performance distribution, and this motivates the resolution to the issue of performance distributability. Albeit in a simple setup, this paper presents an attempt to attacking this problem. Important results are obtained for performance redistribution under both unitary and specified specifications. Constraints are also considered revealing the tight bounds on both nodes dynamics and graph elements for fulfilling the performance distribution and redistribution requirements. Examples are presented for verification of the claims.
\end{abstract}

\section{Introduction}

It has been widely accepted that complex networks encode many biological, technological, and even social systems, and many important results have been disseminated over the past decade [1-6]. Fundamental issues associated with both dynamical properties and control designs for complex networks have been extensively investigated such as network formations [7-9], network propagations [10], network controllability and observability [11-13], and network estimation [14]; collective behaviors such as synchronization [15-17] and consensus and flocking [18-20] have also been analyzed with many important results on conditions for emergences.

Besides the abovementioned research themes, there is yet another important problem to be addressed: the issue of performance distribution. The concept of performance distribution comes from the practical engineering requirements where performance variables are specified with different even contradicting performance indices.
For example, in the newly developed tuned mass dampers, one performance variable is required to be attenuated for vibration isolation, while another one should be significantly increased for energy harvesting purpose [21, 22]. Performance distribution is best exemplified by the unmanned aerial vehicle application where one performance variable is such tuned to harvest energy for self-controlling and self-power [23]. Indeed, such concepts have promising applications in many engineering fields, yet this new requirement has not received enough attention in complex network research. This is not curious since collective behaviors such as synchronization, consensus, or flocking are the main concern in complex networks. In these studies, the objective is usually defined by unitary performance index such as tracking error approaching zero, or a set under persistent perturbation. This is different from performance distribution where one set of performance variables are required approaching zero, while others must be maintained above certain threshold values for efficient utilization (e.g., energy harvesting). 
Consequently the requirement of performance distribution is close to that of multiobjective optimization where more than one objective functions need to be optimized $[24,25]$. Yet, multiobjective optimization problems are usually solved by intelligent algorithms that can often become very complicated for finding compromise solutions. This is particularly true for the design of optimal parameters with constraints. Physical insights are thus lost during the optimization process. In this paper, however, a simple yet powerful method is introduced for attacking the performance distribution and redistribution problems. Important physical insights can be retained when addressing both nominal and constrained designs. Meanwhile, as technology of the future seems to be more intelligent and connected which is strongly featured by smart agents with increasingly complex interactions, it is therefore focusing on the investigation of the effect of topological graph on network performance distribution properties. The structure of the paper is as follows. Section 2 presents problem formulation, while the solutions are given in Section 3. Section 4 considers the problem under distribution specification, while Section 5 further investigates the important problem of performance distribution with parametric constraints. Finally, Section 6 draws the conclusion and discussion which are given in relation to the contribution of the current paper.

\section{Problem Formulation}

Consider a networked system with nonidentical yet linear nodes' dynamics subject to exogenous disturbances:

$$
\dot{x}_{i}(t)=a_{i} x_{i}(t)+\sum_{\substack{k=1 \\ k \neq i}}^{N} s_{i k} x_{k}(t)+s_{i 0} d_{i}(t), \quad i \in[1, N],
$$

where $x_{i}(t)$ is the state variable of the node $i ; a_{i}$ is the corresponding node dynamics, and it is further assumed that $a_{i}$ and $s$ are constants yet different, henceforth representing a heterogeneous network; $s_{i k}$ is the topological interaction among the nodes, and $s_{i 0}$ is the interaction strength with the exogenous signal $d_{i}(t)$. Thus, equation (1) represents a network with $N$ nodes and unity inner coupling $[26,27]$.

To proceed, transform equation into complex domain leading to

$$
\left(s-a_{i}\right) X_{i}=\sum_{\substack{k=1 \\ k \neq i}}^{N} s_{i k} X_{k}+s_{i 0} D_{i}, \quad i \in[1, N]
$$

where $s$ is the complex variable. For ease of presentation, the dependence of $X_{i}(s)$ on $s$ has been omitted. Now, at a frequency $\omega_{0}$, suppose the nodes dynamics are stable; then, (2) becomes an $N$-simultaneous algebraic equations and henceforth $X_{1}\left(j \omega_{0}\right), X_{2}\left(j \omega_{0}\right), \ldots, X_{N}\left(j \omega_{0}\right)$ can be solved for $D_{i}\left(j \omega_{0}\right)$ as follows:

$$
\left[\begin{array}{c}
X_{1} \\
X_{2} \\
\vdots \\
X_{N}
\end{array}\right]=\left[\begin{array}{cccc}
\left(a_{1}-j \omega_{0}\right) & s_{12} & \cdots & s_{1 N} \\
s_{21} & \left(a_{2}-j \omega_{0}\right) & \cdots & s_{2 N} \\
\vdots & \vdots & \ddots & \vdots \\
s_{N 1} & s_{N 2} & \cdots & \left(a_{N}-j \omega_{0}\right)
\end{array}\right]^{-1}\left[\begin{array}{c}
s_{10} D_{1} \\
s_{20} D_{2} \\
\vdots \\
s_{N 0} D_{N}
\end{array}\right] .
$$

Remark 1. The advantage of writing in the matrix representation is that network system dynamics can be captured from frequency domain identification during commission stage, and this is often crucial for large-scale interconnected networks.

To proceed, note that, for a discrete frequency $\omega_{0}$, the exogenous disturbances can be expressed as $s_{i 0} D_{i}\left(j \omega_{0}\right)=$ $C_{i} D\left(j \omega_{0}\right)$, where $C_{i}$ is a complex number representing the gain and phase shift with respect to the signal $d(t)$. With this convention, the frequency responses of $X_{i}\left(j \omega_{0}\right) s$ can be represented with respect to $D\left(j \omega_{0}\right)$ as follows:

$\left[\begin{array}{c}X_{1} \\ X_{2} \\ \vdots \\ X_{N}\end{array}\right]=\left[\begin{array}{cccc}\left(a_{1}-j \omega_{0}\right) & s_{12} & \cdots & s_{1 N} \\ s_{21} & \left(a_{2}-j \omega_{0}\right) & \cdots & s_{2 N} \\ \vdots & \vdots & \ddots & \vdots \\ s_{N 1} & s_{N 2} & \cdots & \left(a_{N}-j \omega_{0}\right)\end{array}\right]^{-1}\left[\begin{array}{c}C_{1} \\ C_{2} \\ \vdots \\ C_{N}\end{array}\right] D$.
Now, denote $X=\left[\begin{array}{c}X_{1} \\ X_{2} \\ \vdots \\ X_{N}\end{array}\right]$ and $C=\left[\begin{array}{c}C_{1} \\ C_{2} \\ \vdots \\ C_{N}\end{array}\right] ;$ define

$S=\left[\begin{array}{cccc}0 & s_{12} & \cdots & s_{1 N} \\ s_{21} & 0 & \cdots & s_{2 N} \\ \vdots & \vdots & \ddots & \vdots \\ s_{N 1} & s_{N 2} & \cdots & 0\end{array}\right]$ and

$\Lambda=\operatorname{blockdiag}\left(\left(a_{1}-j \omega_{0}\right) \cdots\left(a_{N}-j \omega_{0}\right)\right)$. Then, the above equation becomes

$$
X=(\Lambda+S)^{-1} C D \text {. }
$$

Obviously, $G$ is the topological graph and $\Lambda$ defines the nodes dynamics. Further manipulation leads to

$$
X=\left(I+\Lambda^{-1} S\right)^{-1} \Lambda^{-1} C D \text {. }
$$

Equation (6) defines the disturbance distribution property of the nodes for any specific topology graph $S$. It thus provides a foundation for further reshaping of performance distribution for each node. Consequently, the problem of performance redistribution through topology can be posed as follows:

2.1. Problem of Performance Redistribution. Given a performance specification for all the nodes of the complex network, find a topology graph $S$, such that the specification is satisfied; if the specification cannot be satisfied, provide conditions explaining the failure of satisfaction. 


\section{Performance Redistribution}

To proceed, it is envisioned that a given topology graph produces two zones of performance distribution, namely, (1) attenuation zone and (2) enhancement zone. Thus, the above problem of performance redistribution can be recast to reshape the two zones by topology redesign. Without loss of generality, it is assumed that two nodes $X_{1}$ and $X_{2}$ correspond to the two zones, e.g., $X_{1}$ is attenuated and $X_{2}$ is enhanced. Henceforth, the original performance redistribution problem can be reformulated as shaping $X_{1}$ being attenuated and $X_{2}$ being enhanced. That is, the following definition is in force:

$$
\left(I+\Lambda^{-1} S\right)^{-1} \Lambda^{-1} \equiv\left[\begin{array}{ll}
G_{11} & G_{12} \\
G_{21} & G_{22}
\end{array}\right] .
$$

Thus,

$$
\left[\begin{array}{l}
X_{1} \\
X_{2}
\end{array}\right]=\left[\begin{array}{l}
G_{11} C_{1}+G_{12} C_{2} \\
G_{21} C_{1}+G_{22} C_{2}
\end{array}\right] D .
$$

The topology leads to the following disturbance distribution property:

$$
\begin{aligned}
& \left|G_{11} C_{1}+G_{12} C_{2}\right|<1, \\
& \left|G_{21} C_{1}+G_{22} C_{2}\right|>1 .
\end{aligned}
$$

Now, the reshaping of the performance distribution requires

$$
\begin{aligned}
\left|\bar{G}_{11} C_{1}+\bar{G}_{12} C_{2}\right| & >1, \\
\left|\bar{G}_{21} C_{1}+\bar{G}_{22} C_{2}\right| & <1,
\end{aligned}
$$

with redesigned parameters $\bar{G}_{i k}$.

This can be reformulated as the existence of $\Delta_{i k}\left(\bar{G}_{i k}=\right.$ $\left.G_{i k}+\Delta_{i k}\right)$ such that the following is fulfilled:

$$
\begin{aligned}
& \left|\left(G_{11}+\Delta_{11}\right) C_{1}+\left(G_{12}+\Delta_{12}\right) C_{2}\right|>1, \\
& \left|\left(G_{21}+\Delta_{21}\right) C_{1}+\left(G_{22}+\Delta_{22}\right) C_{2}\right|<1 .
\end{aligned}
$$

Obviously, should there be total freedom upon the choice of $\Delta_{i k},(11)$ is always achievable; however, constraints must exist for any nontrivial networks. The most common situation is a symmetric graph. If $S$ is symmetric, so is $\left(I+\Lambda^{-1} S\right)^{-1} \Lambda^{-1}$, implying that $G_{i k}=G_{k i}$. This reduces the number of freedom in $\Delta_{i k}$, and a known representation in $\Lambda$ would further reduce the freedom by the number of $N$. While there are two sets of inequalities in both (9) and (10), two sets of conditions for the unknowns can be derived. And consequently the problem of performance redistributablity is determined by the compatibility of the two sets of conditions for the unknowns to be satisfied. This can be summarized as follows.

Proposition 1. For a symmetric graph, performance redistribution is achievable if and only if the two sets of conditions specified for the unknowns resulting from inequalities (9) and (10) are simultaneously solvable.
Example 1. In the case of two nodes, there are only one unknown variable for a symmetric graph. Assume $\Lambda+S=$ $\left[\begin{array}{cc}a_{1}-j \omega & s_{12} \\ s_{12} & a_{2}-j \omega\end{array}\right]$ which is symmetric. For illustration purpose, it is simply assumed that $C_{1}, C_{2}$, and $C_{3}$ are unities. Then, it can be calculated:

$$
\left(I+\Lambda^{-1} S\right)^{-1} \Lambda^{-1}=\frac{1}{\left(a_{1}-j \omega\right)\left(a_{2}-j \omega\right)-s_{12}^{2}}\left[\begin{array}{cc}
a_{2}-j \omega & -s_{12} \\
-s_{12} & a_{1}-j \omega
\end{array}\right] .
$$

Now, assume that the parameters satisfy

$$
\begin{aligned}
& \left|\frac{a_{2}-j \omega-s_{12}}{\left(a_{1}-j \omega\right)\left(a_{2}-j \omega\right)-s_{12}^{2}}\right|<1, \\
& \left|\frac{a_{1}-j \omega-s_{12}}{\left(a_{1}-j \omega\right)\left(a_{2}-j \omega\right)-s_{12}^{2}}\right|>1 .
\end{aligned}
$$

Thus, the problem transforms to whether there exists $\Delta_{12}=\bar{s}_{12}-s_{12}$ such that the following representations are fulfilled:

$$
\begin{aligned}
& \left|\frac{a_{2}-j \omega-\bar{s}_{12}}{\left(a_{1}-j \omega\right)\left(a_{2}-j \omega\right)-\bar{s}_{12}^{2}}\right|>1, \\
& \left|\frac{a_{1}-j \omega-\bar{s}_{12}}{\left(a_{1}-j \omega\right)\left(a_{2}-j \omega\right)-\bar{s}_{12}^{2}}\right|<1 .
\end{aligned}
$$

Now, (13) and (14) will be satisfied with the following conditions fulfilled, respectively:

$$
\begin{aligned}
& \left|a_{2}-j \omega-s_{12}\right|<\left|a_{1}-j \omega-s_{12}\right|, \\
& \left|a_{1}-j \omega-\bar{s}_{12}\right|<\left|a_{2}-j \omega-\bar{s}_{12}\right| .
\end{aligned}
$$

Or equivalently

$$
\begin{array}{r}
\left(a_{1}-a_{2}\right) s_{12}<\frac{\left(a_{1}-a_{2}\right)\left(a_{1}+a_{2}\right)}{2}, \\
\left(a_{1}-a_{2}\right)\left(s_{12}+\Delta_{12}\right)>\frac{\left(a_{1}-a_{2}\right)\left(a_{1}+a_{2}\right)}{2} .
\end{array}
$$

Obviously, for

(1) $a_{1}>a_{2}$, appropriate values of $\Delta_{12}>0$ will fulfill (16)

(2) $a_{1}<a_{2}$, appropriate values of $\Delta_{12}<0$ will fulfill (17)

That is, $\Delta_{12}$ always exists so that (13) and (14) are simultaneously solvable, excluding $a_{1}=a_{2}$.

Assertion 1. For a two-node network with symmetric graph, performance redistribution is always achievable if and only if $a_{1} \neq a_{2}$.

The above assertion has a profound implication: performance redistributability is not influenced by the element $s_{12}$. However, it should be noted that $s_{12}$ can shape the specific performance distribution level. This is to be looked at in detail below. 


\section{Performance \\ Redistribution under Specification}

The above development only answers the question of feasibility of performance redistribution under unity specification. In practical situations, it is more concerned to be capable of disturbance attenuation up to a certain level. For example, it is required that the disturbance should be redistributed above $6 \mathrm{~dB}$. Thus, (9) and (10) must be reformulated as

$$
\begin{aligned}
& \left|G_{11} C_{1}+G_{12} C_{2}\right|<\delta_{1}<1, \\
& \left|G_{21} C_{1}+G_{22} C_{2}\right|>\delta_{2}>1 .
\end{aligned}
$$

And it is required to reshape the performance to be

$$
\begin{aligned}
& \left|\bar{G}_{11} C_{1}+\bar{G}_{12} C_{2}\right|>\delta_{3}>1, \\
& \left|\bar{G}_{21} C_{1}+\bar{G}_{22} C_{2}\right|<\delta_{4}<1,
\end{aligned}
$$

with redesigned parameters $\bar{G}_{i k}$.

In practice, $\delta_{i}$ can possess simple algebraic relationship such as $\delta_{3}=\delta_{2}, \delta_{4}=\delta_{1}$, and $\delta_{2}=\left(1 / \delta_{1}\right)$. Then, with the redefinition of $\delta_{1}=\delta$, (18) and (19) can be written as

$$
\begin{aligned}
& \left|G_{11} C_{1}+G_{12} C_{2}\right|<\delta, \\
& \left|G_{21} C_{1}+G_{22} C_{2}\right|>\frac{1}{\delta}, \\
& \left|\bar{G}_{11} C_{1}+\bar{G}_{12} C_{2}\right|>\frac{1}{\delta}, \\
& \left|\bar{G}_{21} C_{1}+\bar{G}_{22} C_{2}\right|<\delta .
\end{aligned}
$$

Parallel with the development in the above section for unity specification requirement, the following result is available.

Proposition 2. For a symmetric graph, performance redistribution under specification is achievable if and only if the two sets of conditions specified for the unknowns resulting from inequalities (20) and (21) are simultaneously solvable.

Example 2. In Example 1, further consider the problem of performance redistribution under a specification; then, (13) and (14) are written as

$$
\begin{aligned}
& \left|\frac{a_{2}-j \omega-s_{12}}{\left(a_{1}-j \omega\right)\left(a_{2}-j \omega\right)-s_{12}^{2}}\right|<\delta, \\
& \left|\frac{a_{1}-j \omega-s_{12}}{\left(a_{1}-j \omega\right)\left(a_{2}-j \omega\right)-s_{12}^{2}}\right|>\frac{1}{\delta} .
\end{aligned}
$$

Thus, the problem transforms to whether there exists $\Delta_{12}=\bar{s}_{12}-s_{12}$ such that the following representations are fulfilled:

$$
\begin{aligned}
& \left|\frac{a_{2}-j \omega-\bar{s}_{12}}{\left(a_{1}-j \omega\right)\left(a_{2}-j \omega\right)-\bar{s}_{12}^{2}}\right|>\frac{1}{\delta}, \\
& \left|\frac{a_{1}-j \omega-\bar{s}_{12}}{\left(a_{1}-j \omega\right)\left(a_{2}-j \omega\right)-\bar{s}_{12}^{2}}\right|<\delta .
\end{aligned}
$$

Now, (22) and (23) will be satisfied with the following conditions fulfilled, respectively:

$$
\begin{aligned}
& \left|a_{2}-j \omega-s_{12}\right|<\delta^{2}\left|a_{1}-j \omega-s_{12}\right|, \\
& \left|a_{1}-j \omega-\bar{s}_{12}\right|<\delta^{2}\left|a_{2}-j \omega-\bar{s}_{12}\right| .
\end{aligned}
$$

Or equivalently

$$
\begin{array}{r}
\left(s_{12}-\frac{a_{2}-a_{1} \delta^{2}}{1-\delta^{2}}\right)^{2}<\frac{\delta^{2}\left(a_{1}-a_{2}\right)^{2}}{\left(1-\delta^{2}\right)^{2}}-\omega^{2}, \\
\left(s_{12}+\Delta_{12}-\frac{a_{1}-\delta^{2} a_{2}}{1-\delta^{2}}\right)^{2}<\frac{\delta^{2}\left(a_{1}-a_{2}\right)^{2}}{\left(1-\delta^{2}\right)^{2}}-\omega^{2} .
\end{array}
$$

Unlike the situation in Example 1, the validity of the conditions depends not only on $s_{12}$ but also on the frequency $\omega$ under consideration. Indeed, even the feasibility of the specification itself is constrained by the positiveness of the right hand of the inequality:

$$
\frac{\delta^{2}\left(a_{1}-a_{2}\right)^{2}}{\left(1-\delta^{2}\right)^{2}}-\omega^{2}>0
$$

Manipulation of (27) leads to

$$
\left|\frac{a_{1}-a_{2}}{\omega}\right|>\left|\frac{1}{\delta}-\delta\right|
$$

Consequently, condition (28) actually puts a fundamental constraint on performance redistributability specified by $\delta$. Essentially, the following statement has been proved: for a two-node network with symmetric graph, performance redistribution is achievable only if condition (28) is fulfilled. In the following, it will be shown that this condition is also sufficient.

To proceed, given the satisfaction of (28), it can be deduced from (25):

$$
-\sqrt{\frac{\delta^{2}\left(a_{1}-a_{2}\right)^{2}}{\left(1-\delta^{2}\right)^{2}}-\omega^{2}}+\frac{a_{2}-a_{1} \delta^{2}}{1-\delta^{2}}<s_{12}<\sqrt{\frac{\delta^{2}\left(a_{1}-a_{2}\right)^{2}}{\left(1-\delta^{2}\right)^{2}}-\omega^{2}}+\frac{a_{2}-a_{1} \delta^{2}}{1-\delta^{2}}
$$


or

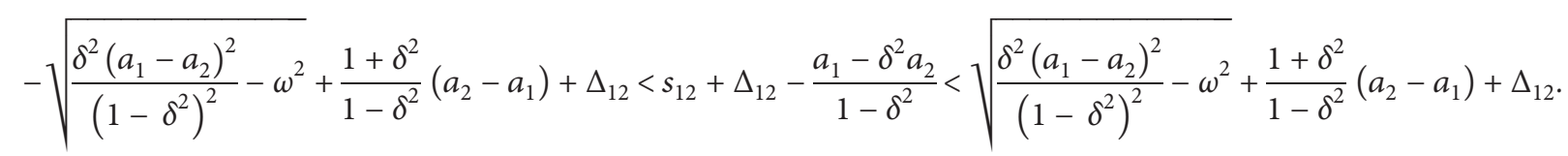

Inequality (30) is the performance redistributability condition inferred from original specification, while (26) is the performance redistributability condition inferred from redistribution requirement which can be simplified as

$$
-\sqrt{\frac{\delta^{2}\left(a_{1}-a_{2}\right)^{2}}{\left(1-\delta^{2}\right)^{2}}-\omega^{2}}<s_{12}+\Delta_{12}-\frac{a_{1}-\delta^{2} a_{2}}{1-\delta^{2}}<\sqrt{\frac{\delta^{2}\left(a_{1}-a_{2}\right)^{2}}{\left(1-\delta^{2}\right)^{2}}-\omega^{2}} .
$$

Thus, (30) and (31) must have at least an intersection set leading to

$$
-2 \sqrt{\frac{\delta^{2}\left(a_{1}-a_{2}\right)^{2}}{\left(1-\delta^{2}\right)^{2}}-\omega^{2}}-\frac{1+\delta^{2}}{1-\delta^{2}}\left(a_{2}-a_{1}\right)<\Delta_{12}<2 \sqrt{\frac{\delta^{2}\left(a_{1}-a_{2}\right)^{2}}{\left(1-\delta^{2}\right)^{2}}-\omega^{2}}-\frac{1+\delta^{2}}{1-\delta^{2}}\left(a_{2}-a_{1}\right) .
$$

To recap, should inequality (32) be satisfied, for $s_{12}$ within (29), original performance distribution (22) and performance redistribution requirement (23) will be feasible simultaneously. The only constraint on satisfaction of (32) is condition (28). Thus, we have proved the following remarkable results.

Assertion 2. For a two-node network with symmetric graph with feasible performance distribution, performance redistribution is achievable if and only if condition (28) is fulfilled, or $\left|\left(a_{1}-a_{2} / \omega\right)\right|>|(1 / \delta)-\delta|$.

Assertion 3. For a two-node network with symmetric graph with $\left|\left(a_{1}-a_{2} / \omega\right)\right|>|(1 / \delta)-\delta|$, performance redistribution is achievable and the required topology $\Delta_{12}=\bar{s}_{12}-s_{12}$ must satisfy (32).
Remark 2. The condition $\left|\left(a_{1}-a_{2} / \omega\right)\right|>|(1 / \delta)-\delta|$ relates performance redistribution specification to nodes' dynamics and frequency, which also answers the important problem of redistributability. Hence, Assertion 2 is a fundamental result for performance redistribution under a general performance specification.

Remark 3. It is now seen clearly that Assertion 1 is a special case of Assertion 2 with $\delta=1$.

As a typical requirement of a $6 \mathrm{~dB}$ redistribution being targeted (hence, $\delta=0.5$ ) with a feasible specification, the above assertions claim that performance redistribution is achievable if and only if $\left|\left(a_{1}-a_{2} / \omega\right)\right|>1.5$. And the required topology $\Delta_{12}=\bar{s}_{12}-s_{12}$ should satisfy

$$
-2 \sqrt{\frac{4\left(a_{1}-a_{2}\right)^{2}}{9}-\omega^{2}}-\frac{5}{3}\left(a_{2}-a_{1}\right)<\Delta_{12}<2 \sqrt{\frac{4\left(a_{1}-a_{2}\right)^{2}}{9}-\omega^{2}}-\frac{5}{3}\left(a_{2}-a_{1}\right) .
$$

\section{Performance \\ Redistribution with Constraints}

As the graph can often represent physical topological connections, e.g., mass-damper-spring systems, the physical parameters are positive that would require the elements of $S$ being positive-definite or negative-definite; meanwhile, the node dynamics in considered systems can be (non) Hurwitzian. Thus, this will further constrain the redistributability of performance. A general discussion can be preceded with detailed classifications, e.g., symmetric graph with positive-definite elements and Hurwitzian nodes dynamics and symmetric graph with negative-definite elements and non-Hurwitzian node dynamics. To clarify and illustrate the 
basic ideas, we shall restrict to the discussion of the situation for symmetric graph with positive-definite elements. The remaining cases can then be deduced by parallel analogy from the development below.

Giving this prelude, the following statement is a straightforward extension from Proposition 2.

Proposition 3. For a symmetric graph with positive-definite elements, performance redistribution is achievable if and only if the feasible $S$ and $\bar{S}$ in (19) and (20), respectively, have elements being simultaneously positive-definite.

The above proposition is best exemplified from the following examples.

Example 3. In Example 2, further consider the constraint $s_{12}>0$; then, for the corresponding $S$ and $\bar{S}$ to be feasible, there must be

$$
\begin{gathered}
-\sqrt{\frac{\delta^{2}\left(a_{1}-a_{2}\right)^{2}}{\left(1-\delta^{2}\right)^{2}}-\omega^{2}}+\frac{a_{2}-a_{1} \delta^{2}}{1-\delta^{2}}<s_{12}<\sqrt{\frac{\delta^{2}\left(a_{1}-a_{2}\right)^{2}}{\left(1-\delta^{2}\right)^{2}}-\omega^{2}}+\frac{a_{2}-a_{1} \delta^{2}}{1-\delta^{2}}, \\
-\sqrt{\frac{\delta^{2}\left(a_{1}-a_{2}\right)^{2}}{\left(1-\delta^{2}\right)^{2}}-\omega^{2}}+\frac{a_{1}-\delta^{2} a_{2}}{1-\delta^{2}}<s_{12}+\Delta_{12}<\sqrt{\frac{\delta^{2}\left(a_{1}-a_{2}\right)^{2}}{\left(1-\delta^{2}\right)^{2}}-\omega^{2}}+\frac{a_{1}-\delta^{2} a_{2}}{1-\delta^{2}} .
\end{gathered}
$$

Thus, should the element be positive, the following inequalities must hold:

$$
\begin{aligned}
& -\sqrt{\frac{\delta^{2}\left(a_{1}-a_{2}\right)^{2}}{\left(1-\delta^{2}\right)^{2}}-\omega^{2}}+\frac{a_{2}-a_{1} \delta^{2}}{1-\delta^{2}}>0, \\
& -\sqrt{\frac{\delta^{2}\left(a_{1}-a_{2}\right)^{2}}{\left(1-\delta^{2}\right)^{2}}-\omega^{2}}+\frac{a_{1}-\delta^{2} a_{2}}{1-\delta^{2}}>0 .
\end{aligned}
$$

Manipulation leads to

$$
\begin{aligned}
& a_{2}^{2}-\delta^{2} a_{1}^{2}+\left(1-\delta^{2}\right) \omega^{2}>0 \\
& a_{1}^{2}-\delta^{2} a_{2}^{2}+\left(1-\delta^{2}\right) \omega^{2}>0 .
\end{aligned}
$$

Together with (28), it is seen that performance redistributability is "severely" restricted by the constraint demanded by positive $s_{12}$. This is summarized as follows.

Assertion 4. For a two-node network with symmetric graph and positive graph elements, performance redistribution is achievable if only if (28), (36), and (37) are simultaneously satisfied.

Remark 4. As a natural consequence, the freedom for allowable node dynamics and even frequency range are also restricted whose Hurwitzianess and domains are actually already determined.

To be specific, consider a $6 \mathrm{~dB}$ redistribution being targeted; then, (28), (36), and (37) read

$$
\begin{array}{r}
a_{1}^{2}-2 a_{1} a_{2}+a_{2}^{2}-2.25 \omega^{2}>0, \\
a_{2}^{2}-0.25 a_{1}^{2}+0.75 \omega^{2}>0,
\end{array}
$$

$$
a_{1}^{2}-0.25 a_{2}^{2}+0.75 \omega^{2}>0
$$

From (38) and (39), it is seen that not only the node dynamics $a_{1}$ and $a_{2}$ but also the allowable frequency range $\omega$ are already prescribed through the requirement of positive $s_{12}$. For example, for $a_{1}=\omega=1$, then $a_{2}$ is restricted to be $(-2.65-0.5) \cup\left(\begin{array}{ll}2.5 & 2.65\end{array}\right)$; yet, for $a_{1}=1$ and $a_{2}=10$, the frequency range is narrowed down to (5.66 6.0), which almost renders the performance redistribution infeasible.

Remark 5. Henceforth, as a final remark, it is noted that the performance redistribution can eventually become unachievable if more constraints exist, e.g., domain restriction to either graph elements or nodes dynamics $\left(a_{i} \in\left[\begin{array}{ll}a_{i 1} & a_{i 2}\end{array}\right]\right)$ and magnitude constraints due to spatial restrictions. Performance distribution and redistribution requirements thus provide tight bounds on both nodes dynamics and graph elements.

\section{Conclusion and Discussion}

The important problem of performance distribution has been disseminated. Important results upon performance distribution under both unity and general specifications have been obtained. Numerical examples have been followed with each claim to clarify the result and better exemplify the illustrations. The contribution of the paper can be best recognized by recasting the discussed problems into the general complex networks research themes: complex networks have been conventionally approached with the consideration of synchronization, consensus, or flocking, where the objective has been defined by the performance index as a tracking error approaching zero or a set. Performance distribution, however, concerns both attenuation and enhancement. This requirement comes from the practical need from the emerging area of vibration energy harvesting, noise cloaking in military submarines, etc. Indeed, applications provide strong motivation to network control theory, and new requirement thus calls upon new theoretical development. 
Therefore, other than providing detailed applicationspecific results, a framework is established for investigation of the new requirement of performance distribution problems. Avenues such as performance distribution and redistribution over a frequency band and nonlinear redistribution can be explored. Such a methodology would thus forge many design methods together for resolution of the important problem of performance distribution for any specific problems associated with practical engineering.

\section{Data Availability}

The data used to support the findings of the study are available from the corresponding author upon request.

\section{Conflicts of Interest}

The authors declare that they have no conflicts of interest.

\section{Acknowledgments}

The authors are grateful for the financial support by the Fundamental Research Funds for the Central Universities (no. NS2020017); Foundation for University Key Teacher by the Ministry of Education of Henan Province (2018GGJS234); Central Military Commission Foundation to Strengthen Program Technology Fund (no. 2019-JCJQ-JJ-347); Central Military Commission Special Fund for Defense Science, Technology \& Innovation (no. 20-163-00-TS-009-096-01); State Foreign Affairs Bureau Fund for Introduction Plan of High-end Foreign Experts (G20200010100); and Aviation Science Fund of ChinaXi'an 631 Research Institute (201919052001).

\section{References}

[1] B. S. Manoj, A. Chakraborty, and R. Singh, Complex Networks: A Networking and Signal Processing Perspective, Pearson, New York, NY, USA, February 2018.

[2] E. Estrada, The Structure of Complex Networks: Theory and Applications, Oxford University Press, Oxford, UK, 2011.

[3] R. Cohen and S. Havlin, Complex Networks: Structure, Robustness and Function, Cambridge University Press, Cambridge, UK, 2010.

[4] N. Ganguly, A. Deutsch, and A. Mukherjee, Dynamics on and of Complex Networks Applications to Biology, Computer Science, and the Social Sciences, Springer, Berlin, Germany, 2009.

[5] M. Newman, A. Barabási, and D. J. Watts, The Structure and Dynamics of Networks, Princeton University Press, Princeton, NJ, USA, 2006.

[6] S. N. Dorogovtsev and J. F. F. Mendes, Evolution of Networks: From Biological Networks to the Internet and $W W W$, Oxford University Press, Oxford, UK, 2003.

[7] S. Rosenfeld, "Mathematical descriptions of biochemical networks: stability, stochasticity, evolution," Progress in Biophysics and Molecular Biology, vol. 106, no. 2, pp. 400-409, 2011.

[8] M. E. J. Newman, "The structure and function of complex networks," SIAM Review, vol. 45, no. 2, pp. 167-256, 2003.

[9] R. Albert and A.-L. Barabási, "Statistical mechanics of complex networks," Reviews of Modern Physics, vol. 74, no. 1, pp. 47-97, 2002.
[10] W. Wang, M. Tang, H. E. Stanley, and L. A. Braunstein, "Unification of theoretical approaches for epidemic spreading on complex networks," Reports on Progress in Physics, vol. 80, no. 3, Article ID 036603, 2017.

[11] Y.-Y. Liu, J.-J. Slotine, and A.-L. Barabási, "Controllability of complex networks," Nature, vol. 473, no. 7346, pp. 167-173, 2011.

[12] N. J. Cowan, E. J. Chastain, D. A. Vihena, J. S. Freudenberg, and C. T. Bergstrom, "Nodal dynamics, not degree distributions, determine the structural controllability of complex networks," PLoS One, vol. 7, no. 6, Article ID e28398, 2012.

[13] J. Ruths and D. Ruths, "Control profiles of complex networks," Science, vol. 343, no. 6177, pp. 1373-1376, 2014.

[14] S. Barbarossa and G. Scutari, "Decentralized maximumlikelihood estimation for sensor networks composed of nonlinearly coupled dynamical systems," IEEE Transactions on Signal Processing, vol. 55, no. 7, pp. 3456-3470, 2007.

[15] F. Dörfler and F. Bullo, "Synchronization in complex networks of phase oscillators: a survey," Automatica, vol. 50, no. 6, pp. 1539-1564, 2014.

[16] Y. Tang, F. Qian, H. Gao, and J. Kurths, "Synchronization in complex networks and its application - a survey of recent advances and challenges," Annual Reviews in Control, vol. 38, no. 2, pp. 184-198, 2014.

[17] A. Arenas, A. Díaz-Guilera, J. Kurths, Y. Moreno, and C. Zhou, "Synchronization in complex networks," Physics Reports, vol. 469, no. 3, pp. 93-153, 2008.

[18] M. Miguel, J. Parley, and R. Satorras, "Effects of heterogeneous social interactions on flocking dynamics," 2018, http:// arxiv.org/abs/11801.03371.

[19] C. Yang and C. Suh, "On the dynamics of complex network," in Proceedings of the ASME 2017 International Mechanical Engineering Congress \& Exposition, Tampa, FL, USA, November 2017.

[20] H. Su and X. Wang, Pining Control of Complex Networked Systems, Springer, Berlin, Germany, 2013.

[21] M. Yuan, K. Liu, and A. Sadhu, "Simultaneous vibration suppression and energy harvesting with a non-traditional vibration absorber," Journal of Intelligent Material Systems \& Structures, vol. 29, 2018.

[22] A. Gonzalez-Buelga, L. R. Clare, S. A. Neild, S. G. Burrow, and D. J. Inman, "An electromagnetic vibration absorber with harvesting and tuning capabilities," Structural Control and Health Monitoring, vol. 22, no. 11, pp. 1359-1372, 2015.

[23] Y. Wang and D. J. Inman, "Simultaneous energy harvesting and gust alleviation for a multifunctional composite wing spar using reduced energy control via piezoceramics," Journal of Composite Materials, vol. 47, no. 1, pp. 125-146, 2013.

[24] J.-H. Cho, Y. Wang, I.-R. Chen, K. S. Chan, and A. Swami, "A survey on modeling and optimizing multi-objective systems," IEEE Communications Surveys \& Tutorials, vol. 19, no. 3, pp. 1867-1901, 2017.

[25] Y. Cui, Z. Geng, Q. Zhu, and Y. Han, "Review: multi-objective optimization methods and application in energy saving," Energy, vol. 125, pp. 681-704, 2017.

[26] J. Lv and G. Chen, "A time-varying complex dynamical network model and its controlled synchronization criteria," IEEE Transactions on Automatic Control, vol. 50, pp. 841-846, 2005.

[27] J. Lv, X. Yu, and G. Chen, "Chaos synchronization of general complex dynamical networks," Physica A, vol. 334, pp. 281$302,2004$. 